\title{
Constrained Decentralized Algorithm for the Relative Localization of Wearable Wireless Sensor Nodes
}

\author{
J. Hamie, B. Denis \\ CEA-Leti Minatec Campus \\ 17 rue des Martyrs, 38054 Cedex 09, Grenoble, France \\ Email: jihad.hamie@cea.fr
}

\author{
C. Richard \\ Universite de Nice Sophia-Antipolis / UMR CNRS 6525 \\ Parc Valrose, 06108 Cedex 2, Nice, France \\ Email: cedric.richard@unice.fr
}

\begin{abstract}
Motion capture might become one key feature of future Wireless Body Sensor Networks (WBSN), allowing new applications such as home activity monitoring, nomadic postural rehabilitation or sportive gesture recording through standard on-body communications. In this context we present herein a stand-alone solution that enables the localization of wearable wireless nodes relatively to a body-strapped Local Coordinate System (LCS). In particular, we consider adapting a Distributed Weighted Multi-Dimensional Scaling (DWMDS) algorithm fed by cooperative inter-node range measurements obtained through e.g., Time Of Arrival (TOA) estimation, where estimated nodes' locations are asynchronously updated based on their neighborhood information. Exploiting further the presence of constantlength radio links, a Constrained solution (CDWMDS) is thus proposed to improve localization accuracy, while reducing traffic and power consumption. Another novelty lies in the initialization step, which somehow benefits from the space-time correlation of nodes' locations under body mobility. Relying on a realistic biomechanical model, we provide preliminary simulation results to illustrate the relative gains observed in comparison with a nominal algorithm setting.
\end{abstract}

\section{INTRODUCTION}

Wireless Body Sensor Networks (WBSNs) are on the verge of fulfilling demanding application needs in a variety of activity domains such as healthcare, security, sports or entertainment. But other innovative solutions based on standalone WBSNs are also expected for next group navigation or motion capture applications [1] - [4]. In the latter context for instance, coarse motion capture capabilities would be still of interest, enabling raw gesture/posture detection for animation purposes (e.g. in gaming, augmented reality, video post-production), emergency and rescue (e.g. detecting elderly people or fire-fighters falling down on the floor), or attitudebased remote sensing and actuating (e.g. for house automation, remote multimedia, industrial control). At the body scale, the relative localization of on-body sensors can then rely on internode range measurements. The latter are typically obtained out of the radio signals transmitted between wireless devices over cooperative peer-to-peer links (e.g. in a mesh network topology). As an example, precise Time Of Arrival (TOA) estimation is usually achieved with the Impulse Radio - Ultra Wideband (IR-UWB) technology [5], which has been recently standardized in the WBSN context [6]. This on-body localization add-on would enable stand-alone and large-scale human motion capture applications, making opportunistic use of the underlying wireless communication means. This could hence represent an appealing alternative to costly, power greedy and geographically restricted video acquisition systems, but most likely at the price of a degraded precision level.

Numerous localization algorithms described in the literature consider centralized resources and synchronous calculi, which are hardly compliant with real-time constraints under realistic human mobility [1], [3] (i.e. estimating all the unknown nodes' locations simultaneously, after relaying inter-nodes measurements to a central coordinator). Moreover, they often underexploit the available potential of mesh topologies by sticking with non-cooperative links (i.e. uniquely with respect to fixed anchors) [2], [4]. Some solutions also necessitate a priori parametric models [4], which may be not that practical, given the unknown location-dependent mobility patterns experienced by on-body nodes (i.e. under non-calibrated arbitrary deployments). Finally, simplified geometric constraints, relying e.g., on the prior knowledge of minimal and maximal feasible distances under constant transmission ranges, have also been introduced [3], assuming a simplified radio behavior.

In this paper, we propose to adapt the Distributed Weighted Multi Dimensional Scaling (DWMDS) localization algorithm [9] into the new body sensor network context. Accordingly, nodes' locations are asynchronously updated with respect to their 1-hop neighbors in a body-strapped Local Coordinate System (LCS), providing better immunity against the latency effects observed within classical centralized schemes and potential adaptability to local nodes velocities (e.g. in terms of refreshment rate). One first point is to feed this algorithm with all the cooperative peer-to-peer distance measurements available in our mesh topology. One more idea is to incorporate links that experience fixed lengths despite body mobility (e.g. between the hand's wrist and the elbow) as geometrical constraints in the positioning problem, leading to a Constrained solution (CDWMDS). This tends to limit the number of required on-line measurements and hence, to reduce over-the-air traffic and power consumption. Finally, 


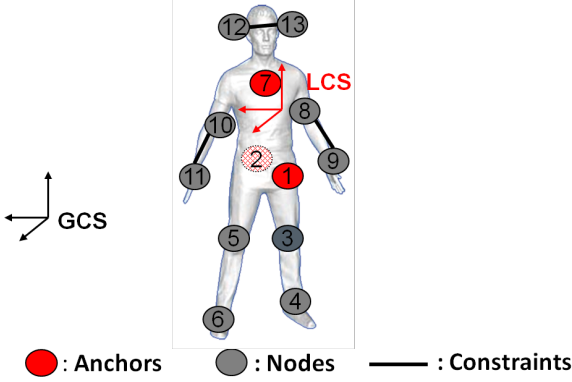

Fig. 1. Typical deployment scenario for the relative localization of on-body wireless nodes (grey circles) with respect to a body-strapped Local Coordinate System (LCS) defined by fixed anchors (red circles).

while updating on-body nodes' locations, we take into account the latest estimates history as prior information, so as to ease convergence and benefit from space-time correlations under body motion.

The paper is structured as follows. In Section II, we introduce the relative localization problem. Section III then presents the core cooperative localization algorithms, including the initial DWMDS algorithm and our new constrained proposal. In Section IV, we describe our simulation set-up and parameters, before showing illustrating results under realistic body mobility. Finally, Section V concludes the paper.

\section{Problem Formulation}

The wireless devices placed on the body can be first classified into two categories. Simple mobile nodes with unknown positions must be located relatively to reference anchors nodes, which are attached onto the body at known and reproducible positions, independently of the body attitude and/or mobility (e.g. on the chest or on the back). A set of such anchors define a time-invariant Cartesian Local Coordinate System (LCS), which remains unchanged under body mobility.

Figure 1 shows a typical deployment scenario, where the LCS is obviously in movement and misaligned relatively to any external Global Coordinate System (GCS). In the following, $\left\{X_{i}(t)\right\}_{i=1, \ldots, n}$ represents the true $3 D$ unknown positions of the $n$ mobile on-body nodes at time $t$, which are to be estimated in the LCS. $\left\{X_{i}(t)=X_{i}\right\}_{i=n+1, \ldots, n+m}$ represents the constant $3 D$ known positions of the $m$ anchors defined into the LCS at time $t$, where $m$ should be at least equal to 3 . Let $\widetilde{d_{i j}}(t)$ be a range measurement available at time $t$ between nodes $i$ and $j$ and let $l_{i j}$ be a constant distance (i.e. constant over body mobility), which will be considered hereafter as a constraint.

The problem that we want to solve is to estimate the dynamic positions of the mobile nodes into the LCS, given all the available range measurements $\left\{\widetilde{d_{i j}}(t)\right\}$ (e.g. derived through IR-UWB TOA estimation [5], [7]) and the known anchors' on-body locations.

\section{LocAliZATION Algorithms}

A. Distributed Weighted Multidimensonal Scaling (DWMDS) Multi-Dimensional Scaling (MDS) is a powerful centralized technique, which enables to retrieve unknown nodes' positions based on a matrix of inter-node distances. One problem is the need for matrix completeness, with a full knowledge of all the pairwise measurements, what is hard to achieve in realistic wireless contexts (e.g. due to connectivity losses or deliberate network topology restrictions). Another problem within such centralized approaches is the latency effect (i.e. the time elapsed between the collection of the required distance measurements and the final delivery of all the positions' estimates), whereas the body gesture can change rapidly during the collection step. Several improvements to the standard MDS have already been provided recently in location-enabled Wireless Sensor Networks (WSNs), e.g. extending the nominal MDS formalism into dynamic tracking contexts (e.g. [8]). However, motivated by the possibility to operate under partial and varying WBAN connectivity, to be more robust against induced latency effects and to benefit from intrinsic asynchronism while localizing the nodes, we thus seek to estimate the nodes' positions using a fully distributed version of the MDS. As described in [9], the Distributed Weighted MultiDimensional Scaling (DWMDS) indeed allows each mobile node $i$ to localize itself by minimizing a local quadratic cost function as follows:

$$
\begin{aligned}
\widehat{X}_{i}(t) & =\underset{\widehat{X}_{i}^{\prime}(t)}{\operatorname{argmax}}\left[\sum_{j=1}^{n} w_{i j}(t)\left(\delta_{i j}(t)-\widehat{d}_{i j}\left(\widehat{X}_{i}^{\prime}(t), \widehat{X}_{j}(t)\right)\right)^{2}\right. \\
& +\sum_{j=n+1}^{n+m} 2 w_{i j}(t)\left(\delta_{i j}(t)-\widehat{d}_{i j}\left(\widehat{X}_{i}^{\prime}(t), \widehat{X}_{j}(t)\right)\right)^{2} \\
& \left.+r_{i}(t)\left\|\widehat{X}_{i}^{\prime}(t)-\bar{X}_{i}(t)\right\|^{2}\right]
\end{aligned}
$$

where $\widehat{X}_{i}(t)$ is a vector containing the estimated $3 D$ coordinates of node $i, \delta_{i j}(t)$ is a so-called observed distance between node $i$ and $j$ at time $t, \widehat{d}_{i j}\left(\widehat{X}_{i}^{\prime}(t), \widehat{X}_{j}(t)\right)$ denotes the synthetic Euclidean distance between $i$ and $j$ built out of the current estimated coordinates $\widehat{X}_{i}^{\prime}(t)$ and $\widehat{X}_{j}(t), w_{i j}(t)$ is a weight, which reflects the connectivity and the accuracy of the range measurement between nodes $i$ and $j$ at time $t$, so that unavailable links are naturally discarded and inaccurate measurements are down-weighted (or reliable neighbors such as anchors could be over-weighted) in the cost function, $\bar{X}_{i}(t)$ is a vector reflecting some prior information about the position occupied by node $i$ at time $t$, while $r_{i}(t)$ quantifies the reliability of such prior information.

As described in [9], at each time $t$, the dynamic equation (1) is iteratively resolved within a few steps $k$. If $\widehat{X}^{(k)}(t)$ is the matrix whose columns contain all the estimated positions at iteration $k$, node $i$ derives its current coordinates update $\widehat{X}_{i}^{(k)}(t)$ as follows:

$$
\begin{gathered}
\widehat{X}_{i}^{(k)}(t)=a_{i}(t)\left(r_{i}(t) \bar{X}_{i}(t)+\widehat{X}^{(k-1)}(t) \mathbf{b}_{i}^{(k-1)}(t)\right) \\
a_{i}(t)=\sum_{j=1}^{n} w_{i j}(t)+\sum_{j=n+1}^{n+m} w_{i j}(t)+r_{i}(t)
\end{gathered}
$$


and $\mathbf{b}_{i}^{(k)}(t)=\left[b_{1}(t), \ldots, b_{n+m}(t)\right]$ is a vector whose entries are

$$
\begin{aligned}
& b_{j}(t)=w_{i j}(t)\left[1-\frac{\delta_{i j}(t)}{\widehat{d}_{i j}(t)} \quad j \leq n, j \neq i\right. \\
& b_{i}(t)=\sum_{j=1}^{n} \frac{w_{i j}(t)}{\widehat{d}_{i j}(t)}+\sum_{j=n+1}^{n+m} \frac{w_{i j}(t)}{\widehat{d}_{i j}(t)} \\
& b_{j}(t)=2 w_{i j}(t)\left[1-\frac{\delta_{i j}(t)}{\widehat{d}_{i j}(t)}\right] \quad j \geq n
\end{aligned}
$$

Note that $w_{i j}$ naturally accounts for partial connectivity. In the nominal embodiment, one localization cycle is assumed completed once that all the mobile nodes have been updated at least once with respect to their 1-hop available neighbors.

\section{B. Proposed Constrained DWMDS}

One first improvement is proposed to adapt the initial DWMDS formulation into the WBSN relative localization context. It consists in taking benefits from trivial geometric specificities of the human body, but without any prior parametric knowledge (i.e. unlike within techniques requiring articulated chains). Fixed-length links identified on the body are introduced as constraints while positioning (e.g. the link between the wrist and the elbow on Figure 1), thus leading to the Constrained DWMDS solution (CDWMDS). More particularly, the basic idea is to substitute the distance $\delta_{i j}(t)=$ $\widetilde{d}_{i j}(t)$ that would be measured between nodes $i$ and $j$ at time $t$ by an approximated version $\delta_{i j}(t)=l_{i j}$ over the same fixedlength link, which is considered as time-invariant independently of the body gesture, moving direction or velocity. Advantageously, during a first pre-calibration phase (already under body mobility), such fixed-length links can be detected and approximated distances can be learnt once for all by averaging repeated instantaneous measurements over a few time stamps, e.g. $l_{i j}=\frac{1}{N_{c a l}} \sum_{t=-N_{c a l}}^{-1} \widetilde{d}_{i j}(t)$, with $t=-N_{c a l}, \ldots,-1$ if the localization procedure is expected to start at $t=0$. One claimed advantage with the proposed enhancement is that no more ranging measurements are required for these links in the steady-state localization regime. Besides localization accuracy considerations, CDWMDS hence leads to a reduction of the number of exchanged packets, and accordingly, a reduction of both latency and energy consumption. Note that alternatively, in case of suspected distance variability during the localization steady-state phase, the average approximation could be periodically recomputed on the wing within a sliding window, i.e. at time stamp $t, l_{i j}(t)=\frac{1}{N_{c a l}} \sum_{t^{\prime}=t-N_{c a l}}^{t-1} \widetilde{d}_{i j}\left(t^{\prime}\right)$.

Another straightforward improvement consists in taking the latest estimated position available for node $i$ at time $t-1$, as a priori information for initialization, i.e. with $\bar{X}_{i}(t)=\widehat{X}_{i}(t-1)$ in equation (2), hence benefitting from the space-time correlation of the true mobile location under body mobility, while speeding up convergence over $k$ at each time stamp $t$.

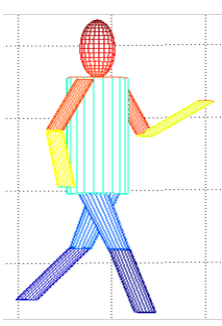

Fig. 2. Biomechanical mobility model based on a piece-wise cylindrical representation.

\section{RESULTS}

\section{A. Simulation Scenario and Parameters}

In our evaluation framework, the overall body mobility in its environment is based on a mixed model, like in [10]. First of all, a macro-mobility Reference Point Group Mobility Model (RPGM) accounts for the body barycenter mobility, where the dynamic reference point as a function of time is chosen as a Random Gauss-Markov process [1]. As for the biomechanical intra-WBAN mobility, it is based on a deterministic piecewise representation of the entire pedestrian [11], where the body extremities are modeled as articulated cylinders. A snapshot of this animated body is shown on Figure 2 for an arbitrary time stamp. This biomechanical model enables the generation of true inter-node distances, whatever the time stamp and whatever the average body position in the environment.

In our scenario, for each random realization, the tested body is moving in a $20 \mathrm{~m} \times 20 \mathrm{~m} \times 4 \mathrm{~m} 3 D$ environment with a constant speed of $1 \mathrm{~m} / \mathrm{sec}$ for $80 \mathrm{sec}$. The network deployment is similar to that presented on Figure 1, where 3 anchors are set at fixed locations and 10 blind mobile nodes must be located relatively to the LCS.

Concerning the radio parameters, we assume that the received power is larger than the receiver sensitivity, which allows peer-to-peer communication links with a worst-case Packet Error Rate (PER) of 1\%, as specified in the IEEE 802.15.6 standard [12]. Then this PER figure is applied onto each packet involved in 3-way protocol transactions [5] to emulate uncomplete ranging transactions (i.e. whenever 1 single packet is lost out of the 3). Medium access issues apart, considering a TOA-based IR-UWB model in a IEEE 802.15.6 mandatory band of $500 \mathrm{MHz}$ centered around $4 \mathrm{GHz}$ [7], additive random ranging errors are conditioned on the Line Of Sight (LOS) or Non Line Of Sight (NLOS) channel configuration at time stamp $t$, as follows:

$$
\begin{array}{llrl}
\widetilde{d_{i j}}(t) & =d_{i j}(t)+n_{i j}(t) & & \text { if LOS } \\
\widetilde{d_{i j}}(t) & =d_{i j}(t)+n_{i j}(t)+b_{i j}(t) & & \text { if NLOS }
\end{array}
$$

where $\widetilde{d_{i j}}(t)$ and $d_{i j}(t)$ are respectively the measured and real distances between nodes $i$ and $j$ at time $t, n_{i j}(t)$ is a centered Gaussian random variable with a standard deviation $\sigma_{n}$, and $b_{i j}(t)$ is a bias term due to the absence of direct path while estimating TOA-based ranges. 


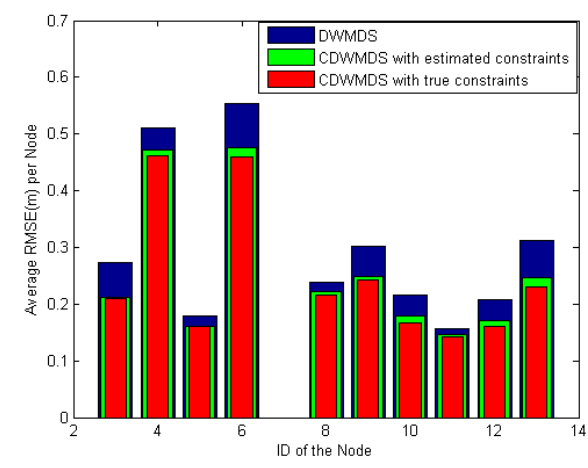

Fig. 3. Root Mean Square Error (RMSE) of the estimated location per on-body node (ID), for various asynchronous and decentralized positioning algorithms: unconstrained (DWMDS - blue), constrained (CDWMDS) with self-calibrated fixed-length ranges (green) and exact fixed-length ranges (red).

Simplifying further the model from [7], our simulations here are carried out using a constant $\sigma_{n}$ of $10 \mathrm{~cm}$, independently of the Signal to Noise Ratio (SNR) at time stamp $t$, but still in the span of typical values observed out of real measurements in [7]. $b_{i j}(t)$ is a positive additive bias in NLOS conditions, which follows a uniform distribution in $[010] \mathrm{cm} . b_{i j}(t)$ is assumed to be constant over one entire walk cycle in first approximation (i.e. $b_{i j}(t)=b_{i j}, \forall t$ ), in compliance with other empirical observations over NLOS portions of trajectory in [7]. In other words, one bias realization can be drawn from the Uniform law once for all at the beginning of each new simulated walk cycle.

Concerning the localization algorithm parameters, three fixed-link constraints are imposed to the CDWMDS algorithm, as shown on Figure 1. We also assume that $w_{i j}(t)$ is equal to 1 (resp. 0) if nodes $i$ and $j$ are connected (resp. disconnected), regardless to neighbor's information reliability (i.e. without soft weighting). $r_{i}(t)$ is also simply taken equal to 1 like in [9]. Finally, localization updates forming a complete localization cycle are performed in average once every $30 \mathrm{~ms}$.

\section{B. Localization Performance}

Simulations have been carried out to illustrate the performance of the proposed algorithm in terms of localization precision. After running consecutive trials of the walk cycle with 100 independent realizations of the ranging errors, we draw the Root Mean Squared Error (RMSE) per node.

As shown on Figure 3, one can expect rather clear benefits from incorporating fixed-length constraints in comparison with the nominal positioning DWMDS, whatever the considered node. Moreover, no significant degradations have been observed after self-learning the fixed-length distances, e.g during a pre-calibration phase, when each constraint is calculated as the mean of the measured distances in an observation window of $9 \mathrm{sec}$ (green bars), in comparison with a genius-aided introduction of the exact fixed-length distances (red bars). The average RMSE per node then spans from $29.5 \mathrm{~cm}$ using DWMDS down to $25.3 \mathrm{~cm}$ and $24.5 \mathrm{~cm}$ using CDWMDS with estimated and true constraints, representing a relative improvement of $14.2 \%$ and $17 \%$ respectively.

\section{CONClusion}

In this paper, we have addressed the problem of motion capture in WBSNs through radiolocation means, based on standard mesh communication links. A decentralized and cooperative DWMDS algorithm, which can asynchronously estimate unknown on-body nodes' locations, has been adapted in the very context. We introduce learnt fixed-length geometric constraints that correspond to time-invariant Euclidean internode distances under body mobility (Constrained DWMDS).

Despite the relative gains observed in terms of average localization error, rather strong limitations are still noticeable, even with reasonably small ranging errors and moderate pedestrian velocity. In particular, a few nodes tend to experience high accelerations and poor Geometric Dilution Of Precision (GDOP) for being placed at the body periphery, causing a certain disparity in the localization performances (i.e. over the nodes) and a still high average localization error, most likely due to error propagation. These very preliminary results suggest to investigate unilateral censoring and scheduling of these nodes while updating the estimated mobile positions.

\section{ACKNOWLEDGMENT}

This work has been carried out in the frame of the CORMORAN project, which is funded by the French National Research Agency (ANR) under the contract number ANR-11INFR-010.

\section{REFERENCES}

[1] E. Ben Hamida, M. Maman, B. Denis, and L. Ouvry, "Localization performance in Wireless Body Sensor Networks with beacon enabled MAC and space-time dependent channel model,' in Proc. IEEE PIMRC'10, pp.128-133, Istabul, Sept. 2010.

[2] H.A. Shaban, M.A. El-Nasr, R.M. Buehrer, "Toward a Highly Accurate Ambulatory System for Clinical Gait Analysis via UWB Radios," IEEE Trans. on Information Technology in Biomedicine, vol.14, no.2, pp.284291, March 2010.

[3] M. Mhedhbi, M. Laaraiedh, and Bernard Uguen, "Constrained LMDS Technique for Human Motion and Gesture Estimation," in Proc. WPNC'12, Dresden, March 2012.

[4] Z.W. Mekonnen, E. Slottke, H. Luecken, C. Steiner, and A. Wittneben, "Constrained Maximum Likelihood Positioning for UWB Based Human Motion Tracking," in Proc. IPIN'10, pp.1-10, Zurich, Sept. 2010.

[5] Z. Sahinoglu, S. Gezici, and I. Guvenc, "Ultra-Wideband Positioning Systems: Theoretical Limits, Ranging Algorithms, and Protocols", Cambridge University Press, 2008.

[6] K.S. Kwal, S. Ullah, N. Ullah, "An Overview of IEEE802.15.6 standard", in Proc. ISABEL'10, Rome, Nov. 2010.

[7] J. Hamie, B. Denis, R. D'Errico, C. Richard, "Modeling of IntraBAN Ranging Errors Based on IR-UWB TOA Estimation,' in Proc. BodyNets'12, Oslo, Oct. 2012.

[8] G. Destino, D. Macagnano, G. Abreu, B. Denis, L. Ouvry, "Localization and Tracking for LDR-UWB Systems", in Proc. IST Mobile and Wireless Communications Summit'07, Budapest, July 2007.

[9] J.A. Costa, N. Patwari, and A. Hero III, "Distributed WeightedMultidimensional Scaling for Node Localization in Sensor Networks", ACM Transactions on Sensor Networks, vol. 2, no. 1, pp. 39-64, Feb. 2006.

[10] M. Maman, F. Dehmas, R. D’Errico, and L. Ouvry, 'Evaluating a TDMA MAC for Body Area Networks using a Space-Time Dependent Channel Model,' in Proc. IEEE PIMRC'09, pp. 2101-2105, Tokyo, Sept. 2009.

[11] I. Pantazis, "Tracking Human Walking using Marg sensors", Master's Thesis, Naval Postgraduate School of Monterey, CA, 2005.

[12] http://www.ieee8012.org/15/pub/TG6.html.

[13] R. D'Errico, L. Ouvry, 'Time-variant BAN Channel Characterization," in Proc. IEEE PIMRC 2009, pp. 3000-3004, Tokyo, Sept. 2009. 